UDC 004.724

DOI: $10.30748 /$ nitps.2021.42.15

\author{
Nooh Taha Nasif
}

AL-Nukhba University College, Baghdad, Iraq

\title{
USING STATISTICAL TRAFFIC ANALYSIS TO CALCULATE THE CONFIDENTIAL MEANS OF INFORMATION TRANSMISSION
}

The article considers the modeling of security problems in the Internet as stochastic systems. This allows you to find flaws in existing security systems and offer new solutions. Studying the vulnerabilities of existing security tools can prevent cyber-attacks from taking advantage of weak systems. New, flexible and adaptive security schemes are necessary for emerging security threats elimination. A hybrid network security scheme, including intrusion detection systems and baits, scattered throughout the network is proposed. This combines the advantages of two security technologies. Honeypot is an activity-based network security system that can be a logical addition to the passive detection policies used by the IDS. This integration forces us to balance the safety indicators compared to costs, planning the operation of the device for the proposed system. Formulation of planning problems as a decentralized partially observable Markov decision-making process (DEC-POMDP) allows to make decisions in a distributed manner on each device without the need of centralized management.

Keywords: security in the system, intrusion detection system, traffic analysis.

\section{Introduction}

Researchers have conducted extensive work to provide reliable and confidential means of information transferring between parties who want to remain anonymous. Partly the reason for this is that it concerns not just the content of the message being transmitted, but important information can also be identified by the identities of the communicating parties. Many anonymity systems with a small delay appeared, such as Tor [1], JAP [2] and I2P [3], etc. Despite the fact that efforts are directed at anonymity in communication, the effectiveness of traffic analysis was shown in violation of the anonymity of these systems. Most anonymity systems are implemented using mixing networks. However, in [4] it is shown, that the majority of existing mixing networks do not work with traffic analysis based on correlation. Traffic analysis was used as means of cryptanalysis, but now it is usually considered as the basis of communication intelligence [5].

As distinct from cryptanalysis, which attempts to crack complex encryption, traffic analysis ignores the content of the message and displays useful information from the metadata associated with the message. It was shown, that the properties of network traffic (such as dimensions and time of package), which remain observable after encryption, show valuable information. As an example, in [6] attack traffic analysis on the body area sensors network (BASN), used for remote patient monitoring is presented. By the frequencies analyze used by wireless radio link between the sensors, they can identify the type of medical sensors, and this information will further detect the patient's health problems.

\section{Statement of basic materials}

\section{Research objective}

We concentrate on the Tor network, Because it is one of the most popular anonymity system around the world. We started a temporary traffic check with Tor. Consider the scenario in which the client interacts with the server via Tor. Assuming, that communication protocol, used by the client can be represented by a hidden Markov model (HMM), we can derive a model that is an exact representation of the basic protocol, using the time information collected on the server side. The proposed confidence model approach is applied to the attack experiment to determine the size of the data needed to build a statistically significant protocol representative.

HMM analyze real-world problems. If for HMM generation an insufficient amount of observational data is used, the model will not be the main process. Existing methods suggest that observations completely reflect the underlying process. The size of the training data often happens to be not enough large for adequately capturing all the statistical dependencies in the system. Therefore, it is important to know the level of statistical significance for the constructed model representing the underlying process, and not just a set of tests.

In this work we construct minimal entropy HMM directly of the observations sequence [7]. We determine whether the observational data and the constructed model fully express the underlying process with a given level of statistical significance. We use statistics process to calculate the upper estimate of the necessary samples number to ensure that the model has a specified significance level, i.e. model confidence. 


\section{Problem state}

Security became a serious problem in computer networks. Intrusion detection system (Intrusion Detection System, IDS) track real-time network traffic for suspicious behavior and alert the system administrator. In particular cases IDS can also be programmed to respond. However, traditional passive security technologies, such as intrusion detection and firewalls, no longer meet the requirements of complex network security. IDS require signatures of existing attacks and often cannot detect unknown hostile actions, while new attacks always emerge. Taking into consideration these limitations, IDS should not be the only security tool [8].

Many researchers suggested combining different security strategies to get more efficient capacity. We unite IDS with honeypots. Attractant is an active protection system designed to overcome IDS deficiencies. These security resources are intended for verification or compromise. Honeypots distract and distract opponents from important services and computers in the network. As a result, the threat imposed on the real goal is somewhat mitigated. At the same time, honeypots lower false positive (FP) IDS rates, watching captured attackers and their attack methods. Appeal characteristics make honeypot a useful addition to IDS. The basic idea is to deploy baits that lure malicious traffic by emulating services and operating systems.

Full awareness of the choice consequences is important for making reasonable decisions. In real applications such information is often not available or is not a deterministic probability distribution over the set of possible results. Knowledge of the current situation is also typically incomplete. Markov decision process (MDP) It is suitable for modeling problems in which we are not sure in the choice consequences. Partially observable Markov decision process (POMDP) are taken, when both of the above uncertainties are present.

POMDP are control systems that cannot be directly observed. They model stochastic media with hidden states. Summarizing MDP and assuming greater uncertainty, POMDP provide a more powerful formalism for realistic problems modeling, especially for managing systems with noisy data or limited sensitivity. A number of problems in industry, business, military and social sciences can be modeled as POMDP, including eliminating network problems, searching for moving targets, autonomous robot design, marketing, medical diagnostics, etc.

\section{Key definitions}

\section{Probability state}

The set denoted as $\Omega$, is known as sample selection space. Each $\omega \in \Omega$ result is a full description of the final experiment state. Any subset $E$ sample space $\Omega$ is called an event. We define a real function $P$ of $\Omega$ subsets, which satisfies the following three axioms:
1. $0 \leq P(E) \leq 1$.

2. $P(\Omega)=1$.

3. $P\left(E_{i} \cup E_{j}\right)=P\left(E_{i}\right)+P\left(E_{j}\right)$

for disjoint events $E_{i}$ and $E_{j}$, i.e. $E_{i} \cap E_{j}=\varnothing$ each time, when $i \neq j$.

$P(E)$ is called the event probability $E$. Formally, a probability space is defined for each random experiment by a triple $(\Omega, F, P)$, where $F \subseteq 2^{\Omega}$ ( $2^{\Omega}-$ multiple power $\Omega$ ) is a space of events.

\section{Probability States}

Probability states are one of the key tools in probability theory for uncertainty justification. Consider a probability space $(\Omega, F, P)$, let's assume, that an event $E \in \Omega$ occurred, How does this affect the probability of other events? Possibility of $F$ event, arising under the state that $E$ occurs, is defined as

$$
P(F \mid E)=\frac{P(F \cap E)}{P(E)},
$$

where $P(F \cap E)$ - joint probability events of $F$ and $E$, i.e. probability of the fact, that these two events will occur joint simultaneously. Two events are called independent if and only if, when $P(E \cap F)=P(E) P(F)$ or, what is the same, $P(E \cap F)=P(E)$, according to (1). That is why, in order to say, that $E$ and $F$ events are independent, it is enough to say, that $E$ observation has no influence on the $F$ probability.

Now we represent three basic but important rules for managing state probabilities of joint probabilities. The first is known as a product rule.

\section{Product rule}

$$
P(F \cap E)=P(F \mid E) P(F) .
$$

Joint probability of events $E$ and $F$ is the probability product of the fact, that $F$ occurs, and the probability, that $F$ will occur, if $E$ happens.

The second rule that we are going to introduce is the chain rule. It can be considered as a generalization of the product rule to several events.

Chain rule. Repeating the product rule:

$$
\begin{gathered}
P\left(\bigcap_{i=1}^{n} E_{i}=\right. \\
\left.\cap E_{2}\right) \ldots P\left(E_{n}\right) P\left(E_{2} \mid E_{1}\right) P\left(E_{3} \mid E_{1} \cap\right) .
\end{gathered}
$$

The last rule covered here is the formula usually used when trying to get an expression from the probability state of one event given by another, namely the Bayesian rule.

Bayesian rule. Suppose now that an event has occurred, we want to find $F$ probability. Using the product rule in (2), both direct and reverse, we have 


$$
P(F \mid E)=\frac{P(E \mid F) P(F)}{P(E)} .
$$

\section{Random Variables}

In practice, we are mainly interested in some real functions of the results, not the result. These functions, defined on the sample space, are known as random variables. More formally random variable is $X: \Omega \rightarrow R$ function. Random variables play an important role in probability theory. If a random variable takes a finite or countable number of possible values, it is called a discrete random variable. There is an opposite type of random variables that take a continuum of values and are known as continuous random variables. One of the most spread probability measures of random values is the cumulative distribution function $(c d f)$, which is $F_{X}: R \rightarrow[0,1]$ function, defined as

$$
F_{X}(x)=\operatorname{Pr}\{X \leq x\} .
$$

Compared to $c d f$ more often used probability measure for a discrete random variable $X$ is its mass probability function ( $p m f) p(x)$ and is defined as

$$
p_{X}(x) \triangleq \operatorname{Pr}\{X=x\}
$$

with properties

$$
\begin{aligned}
& -0 \leq p_{X}(x) \leq 1 ; \\
& \text { - } \sum_{x \in \Omega} p_{X}(x)=1 .
\end{aligned}
$$

For continuous random variables, if $c d p$ is everywhere isomorphic, we define the probability density function ( $p d f$ )as a derivative of $c d f$, i.e.

$$
f_{X}(x) \triangleq \frac{d F_{X}(x)}{d x}
$$

There are two most common descriptive values that we want to know about a random variable: the expected value and the variance. Expected value, also known as the average of $X$ discrete random variable, denoted as $E(X)$, is defined by the formula:

$$
E(X) \triangleq \sum_{x \in \Omega} x p_{X}(x)
$$

Dispersion of a random variable $X$ is distribution deviation measure $X$ from the expected value and is formally defined by the formula:

$$
\operatorname{Var}(X) \triangleq\left[(X-E(X))^{2}\right] .
$$

In addition, the standard deviation $X$, denoted as $\sigma$, is equal to $\sigma=\sqrt{\operatorname{Var}(X)}$.

Markov model is a stochastic process that passes through a set of states over time, when the probability of a transition to any of the other states depends only on the current state.

Let's consider the process that is observed at discrete time points $t=1,2, \ldots$, which will be called steps. At every point in time $t$ the state of the process is de- noted as $S_{t} . S_{t}$ takes values from a finite or countable set $S=\{1,2, \ldots, N\}$, which we call the states space. If $S_{t}=n$ is a process in $n$ state at the time $t$. For each $s, s^{\prime} \in S$ we assume, that every time, when the process is in the state $s$, there is a stationary probability $P_{S s^{\prime}}$, that the process will be in the state $s^{\prime}$. I.e, $P_{s s^{\prime}}$ does not depend on time and any previous states. Such a stochastic process is known as the Markov chain.

\section{Model certainty}

In this work we offer a solution for finding the model certainty. Proposed models will correspond to the sets of training sets. A more important question is whether the learning data completely represent modeling process. Nevertheless, model certainty receives less researchers attention than the model possibility. Most of the previous model evaluations are performed by comparing the results of the constructed model for data verification [9-10]. In [11] a set of model confidence was introduced in order to select the best model with a given confidence level, based on the assumption that there is a set of constructed models.

It shows how one can measure the confidence of a model for systems with a finite number of states and stationary transition probabilities. This approach is similar to work [12]. It uses the approximation position and assumes that the failure probabilities tend to zero with an increase in the number of samples to infinity. During long time term with many large samples this approximation is valid when the probabilities are much smaller than the number of samples. In our work we assume that the probabilities are stationary, but we may not have enough samples for approach. We also calculate the amount of data needed for ensuring that all transitions with a probability exceeding a certain value are statistically adequately included into the model. From this information we obtain models confidence.

Most existing methods for HMM building require an initial model structure that is used as a starting point for the model parameters estimation. To build a Markov model without a priori structural information we extend the Causal State Splitting Reconstruction (CSSR) algorithm. Suppose that we are given sufficient $D$ length training data over a finite $O$ alphabet. Shalizi Approach [7] finds statistically significant groups of training data that correspond to HMM states. It is performed by probability states analyzing for a data window that slides on the learning data. This data window gradually increases from the size of two to the originally known $L$ maximal window size. $L$ parameter expresses the maximal characters number, which are statistically relevant to the next symbol in the sequence.

Although the Shalizi approach allows us to build HMM without an a priori knowledge of the structure, their implementation is based on two critical assump- 
tions: optimal $L$ value was known; in the input observation sequence there was more than enough data. When developing a mathematical algorithm for solving a real problem it is always preferable and even necessary to simplify assumptions to make implementation feasible in practice. Later, an algorithm [13] was developed in order to show, how to automatically find the optimal value $L$, known as HMM output algorithm with zero knowledge. This resolves the first assumption and leaves us only the size of the training data set that needs to be defined.

Because there are small differences between HMM, constructed using CSSR and standard model, defined above, before obtain further insight into CSSR, we'll take a closer look at the specific type of HMM generated by CSSR, as well as the improved algorithm.

HMM, generated by CSSR are known as deterministic HMM and can be denoted as 4-tuple $\langle G=S, O, \Lambda, \zeta\rangle$, where $S$ - finite set of states, $O-$ finite set of observations, $\Lambda \subseteq S \times O \times S$ - transition ratio, and $\zeta: \Lambda \rightarrow[0,1]$ - such a probability function that

$$
\sum_{o^{\prime} \in O, s^{\prime} \in S} \zeta\left(s, o, s^{\prime}\right)=1 \forall s \in S .
$$

Deterministic HMM characteristic is fully reflected in (10). In words, taking into consideration the current state $s$ and observation after the transition. The next $s^{\prime}$ step is determined. The property of a deterministic model can be expressed as follows for each $s \in S$ :

$$
\zeta\left(s, o, s^{\prime}\right)= \begin{cases}P_{s}^{s^{\prime}}=P_{s}^{o}, & \text { if } P_{s}^{s^{\prime}} \neq 0 \text { and } P_{s}^{o} \neq 0, \\ 0, & \text { if } P_{s}^{s^{\prime}}=0 \text { or } P_{s}^{o}=0 .\end{cases}
$$

Due to this characteristic it is possible to more accurately represent deterministic HMM. On the fig. 1 SHMM and an instance of deterministic HMM of the same structure (deterministic HMM with the same number of states and observations) are shown. As distinct from SHMM, in which there is no deterministic relationship between the state and its output, taking into consideration the previous state, each deterministic HMM is assigned with one observation. Moreover, the representation provides us with one more interpretation of the deterministic characteristic, which will not be more than one outgoing transition associated with the same observation for each state.

Recall that the standard model has two sets of random processes: states and outputs. In order to correspond to [7], we use this alternative model, i.e. deterministic HMM, with only one set of random processes. In deterministic HMM, as shown in (10), transitions between states directly correspond to output symbols. The process, developed in [7], creates state computers, that are determined in the transition symbol, i.e. where each symbol is reflected no more than for one transition, leaving the state.

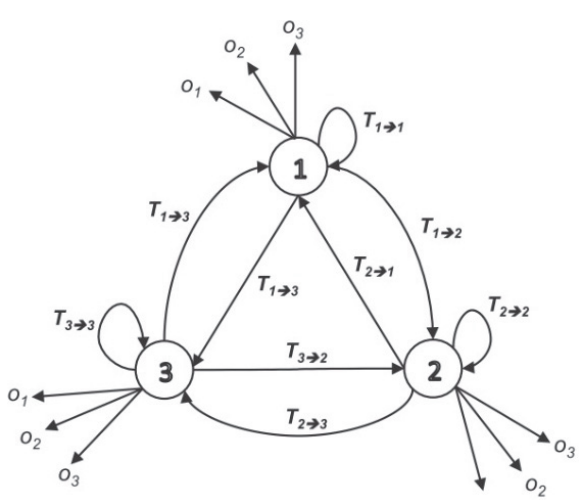

a

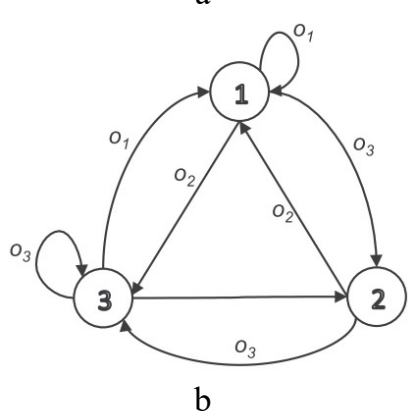

Fig. 1. Three-dimensional observation of HMM and a copy of its deterministic analogues

Let $O-$ be a finite set of symbols or alphabets, representing the observation system space. If $y-$ sequence in $O$, then we write $y^{D}=O_{1} O_{2} \ldots O_{D}$ for $y$ element designations. Let's assume, that $y^{D}$ is the symbolic output of unknown system. We also assume that this system can be represented as a Markov chain, and the probabilities of transitions between states remain unchanged in time.

Our work is an extension of the CSSR [7] algorithm, which describes the structure of the state and transition, $y^{D}$ training data and the size of $L$ data window. This output model is the minimum estimate of the entropy of a true base process. In this approach, each state is associated with a set of subsequences $y^{D}$ with the same probability probability states over the next character generated by the process. In such a way the definition of states allows the system to tolerate noise and uncertainty in the observations sequence and continue to support the system deterministic behavior. The algorithm has useful information-theoretic properties, since it maximizes mutual information between states and output symbols, minimizing the remaining uncertainty (entropy).

Created Markov chain is irreducible with all transition states removed from the model.

HMM output algorithm is simple. We are provided with $y^{D}$ sequence and a priori value $L \in N$ is known. [13] shows, as $L$ value can be set as a part of the HMM training process can be set as, in such a way that HMM 
can be studies without prior knowledge, While the base system has a finite number of states, and the transition probabilities are stationary. With values $l$, increasing from 0 to $L$, we identify the set of $W$ sequences, which are subsequences of $y$ and have $l$ length. (If $l=0$, then an empty line is treated as $y$ subsequence.) We calculate the probability states of the next character after each $x \in W$, using the data provided in $y$, and divide the subsequences in accordance with these probabilities. Let $\#(x, y)$ to be a number of times, when $x$ sequence is observed as $y$ subsequence, probability states distribution to the next input symbol $o$ is given by the formula

$$
f_{o x \mid y}(o)=\operatorname{Pr}(o \mid x, y)=\frac{\#\left(x_{o}, y\right)}{\#(x, y)} .
$$

These sections become states in the output HMM. If the states already exist, we compare $f_{o x \mid y}(o)$ with probability states of existing states, denoted by $f_{s \mid y}(o)$. This is the probability that the system is in a state $s$, that the next observed symbol will be $o$. For calculation of $f_{S \mid y}(o)$ we can use the following formula.

$$
f_{S \mid y}(o)=\operatorname{Pr}(o \mid s, y)=\frac{\sum_{x \in S} \#\left(x_{o}, y\right)}{\sum_{x \in S} \#(x, y)} .
$$

We add $x$ to this state, if probability states are statistically identically managed. If the sequence $x$ can be added to many states, the state with the best choice is selected. The distribution can be performed using $\chi^{2}-$ compatibility with a certain level of reliability. The trust level selects the error rate of the $I{ }^{-}{ }^{\text {st }}$ type. Upon completion of the state formation they are further divided to ensure that the intended model has a deterministic transition relation with a respect to the symbols. Reconstruction combines states, when possible, in order to avoid the creation of an unnecessarily large number of states.

The complexity of the HMM output algorithm is $O\left(|O|^{L+1}\right)+O(D)$, where $|O|-$ alphabet size, and $L$ - the considered maximum length of a subsequence. Taking into consideration $y$ symbols flow, $D$ fixed length, from $O$ set of algorithm is linear at the length of the input data set but is exponential in the size of the alphabet.

Let's notice, that [13] estimates probability states by analyzing the outputs grouped sets from a random process. Until the assumption that the amount of training data takes place, the law of large numbers dictates that this is asymptotically true. We provide a criterion for determining whether an HMM corresponds to an adequate data flow [14]. Another critical assumption is that the set of observations $O$ includes all observations.
Note that CSSR [7] estimates probability states by analyzing the grouped sets of outputs from the stochastic process. Until the assumption that the amount of training data is sufficient, the law of large numbers dictates that this is almost certainly true. This would not be the case if only one sample of a small output line was considered. [13] shows, as $L$ value can be established as part of the HMM training process in such a way, that HMM can be studied without a prior knowledge, if the base system has a finite number of states and the transition probabilities are stationary.

First we put forward the following proposal.

Proposal 1. Taking into consideration HMM and $y^{D}$ observations sequence, порожденных из НММ. Let's consider the $s \in S$ state and the outbound transfer $\delta$ from the state $s, P(\delta)$ - the probability that the transition $\delta$ happened, we have

$$
P(\delta)=\pi_{s} \cdot \gamma_{s}^{\delta}
$$

where $\gamma_{s}^{\delta}$ denotes transition probability $\delta$, taking into consideration, that currently the process is in the state of $s$. For any $\eta \in(0,1)$ such number $0<\varepsilon \leq \pi_{s}$ exists, than for any $\delta$ transition with $P(\delta) \leq \varepsilon$ possibility of the fact, that $\delta$ transition is not determined by $y^{D}$, more, than $\eta$. Relationship between $D, \eta$ and $\varepsilon$ are given by the formula

$$
0<\varepsilon \leq \pi_{s}\left(1-\eta^{\frac{\varepsilon}{\pi_{s} D}}\right),
$$

where $\pi_{s}$ is the asymptotic probability of the state $s$.

Proof. Consider the outbound transition $\delta$ out of $s$ state with $P(\delta)=\varepsilon$, where $0<\varepsilon \leq \pi_{s}$. When visiting a state $s$ random value, representing the emerge of $\delta$ transition, follows the Bernoulli distribution with success probability

$$
p=\gamma_{s}^{\delta}=\frac{\varepsilon}{\pi_{s}}
$$

Let's assume, that $D$ is enough large, estimated number of times, when $s$ state is visited in $y^{D}$, is equal to $D_{s}=\pi_{S} D$. Thus, the probability that $\delta$ transition is not included into $y^{D}$,

$$
(1-p)^{D_{s}}=(1-p)^{\pi_{s} D}
$$

If this probability is bounded from below by $\eta$, we have

$$
(1-p)^{\pi_{s} D}>\eta \text {. }
$$

From the previous equation we have:

$$
p<1-\eta^{\frac{1}{\pi_{s} D}} .
$$

Substituting equation (16) into equation (19), we obtain: 


$$
\varepsilon<\pi_{S}\left(1-\eta^{\frac{1}{\pi_{s} D}}\right) .
$$

This completes the proof.

If $\eta \rightarrow 1, \varepsilon \rightarrow 0$, but at any $\eta<1, \varepsilon \neq 0$. The expression in equation (20) can fully reflect relationship between volume of the training data and uncertainty of the model relatively to the transmissions, which probability meet a certain limitation.

In details

1. at any fixed length of the observation sequence $y^{D}$, larger $\eta$ gives a lower value $\varepsilon$.

2. If we fix the value $\eta$ and increase $D$, then $\varepsilon$ will exponentially decay with $D$.

3 . Finally, in order to maintain a fixed $\varepsilon$ exponential growth is necessary $D$ towards $\eta$.

This analysis gives a boundary value for any of these three variables, when the remaining two are fixed

$$
\varepsilon=\pi_{s}\left(1-\eta^{\frac{1}{\pi_{s} D}}\right) .
$$

This understanding is used below, choosing statistical tools to determine when this threshold value is met. We will also show that for the whole model there will be one threshold value that can check this property for all states.

Let $K_{s}$ be a set of detected outgoing transitions from the state $s$. As an example, we use the model on the fig. 2, a, $K_{1}=\{(1, A),(1, C)\}$. Let $U_{s}$ be a set of unobservable outgoing transitions from $s$. For the model on the fig. 2 , b $U_{1}=\{(1, B)\}$ and $U_{2}=\{(2, B),(2, C)\}$. It's obvious that $K_{s} \cap U_{s}=\varnothing, \forall s \in S$. Now we collect more test data for passing the model. The current model is incomplete after the transition $\delta \in \bigcup_{s \in S} U_{S}$. According to the proposal 1 , we can never be sure that $U_{s}=\varnothing$. We can express maximal possibility of $\varepsilon_{s}$, that transition to $U_{s}$ will take place as follows:

$$
\varepsilon_{s}=\max \left(\varepsilon_{s}^{1}, \varepsilon_{s}^{2}, \ldots, \varepsilon_{s}^{\left|U_{s}\right|}\right)
$$

and for all states we define $\varepsilon^{*}$ as follows:

$$
\varepsilon^{*}=\max \left(\varepsilon_{1}, \varepsilon_{2}, \ldots, \varepsilon_{|S|}\right) .
$$

In such a way, $\varepsilon^{*}$ represents the maximum probability of the transition of all unobserved transitions. Our goal is to determine the total number of samples $D$, such, that $\varepsilon^{*}$ is less than $\varepsilon$ user threshold. This means, all outgoing transitions with probabilities exceeding $\varepsilon$ for all states, must be present in the model constructed with the output character line $y^{D}$.
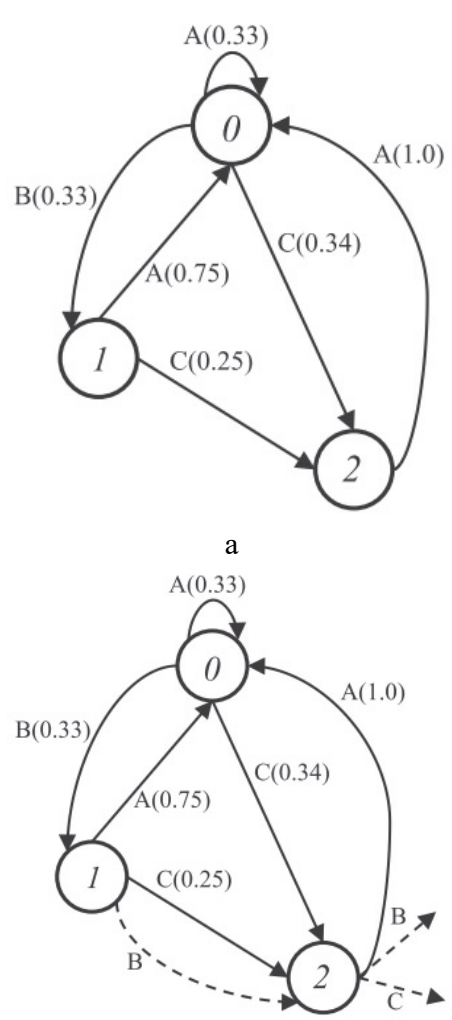

b

Fig. 2. An example of known and unknown transitions for the alphabet $O=\{A, B, C\}$. Solid transitions are

known and probabilities, calculated from the input data are set. Dotted transitions are possible, but can hardly be found in the training data set. a - Assumed HMM; b - Actual model

Therefore, a larger value $\varepsilon$ reduces the amount of data, necessary to make sure that $y^{D}$ Includes any transition with a probability of at least $\varepsilon$, but increases the risk $U_{s}=\varnothing, \forall s \in S$. A smaller threshold reduces this risk, but may require a significant amount of data.

Suppose that we have built HMM using the output algorithm HMM with zero knowledge [13] from the sequence of observations $y^{D}$. Let's consider the transition $\delta$ with probability $\varepsilon$. Without generality limiting, we assume that $\delta$ is a transition from the state $s$ and $0<\varepsilon \leq \pi_{s}$. If we use $\gamma_{s}^{\delta}$ to denote a state probability $\delta$, taking into account that the system is currently in the state of $s$, from equation (16) we have:

$$
\gamma_{s}^{\delta}=\frac{\varepsilon}{\pi_{s}}
$$

where $\pi_{s}$ is asymptotic probability of a state $s$ and is given by the formula:

$$
\pi_{s} \approx \frac{n_{s}}{D}
$$

where $n_{s}$ - number of times, when the state $s$ is introduced during observation $y^{D}$. 
For $s$ states we use $z$-test [15], in order to define, whether the proposed model will include all transitions with probabilities of at least $\gamma_{s}^{\delta}$ with the desired value. $z$-test also finds minimal quantity of training samples, necessary for $\delta$, which should be detected with this given significance level. As mentioned above, the constructed Markov chain is irreducible.

The next step is to define test statistics for $s$ states. Let $X_{S}^{U}$ be a random variable, following the Bernoulli distribution with probability of $p_{s}$ success, we use $X_{S}^{U}$ to describe the emerge of an unobserved transition to a process that is currently in a state. We can test the null hypothesis $H_{0}: p_{s}=\gamma_{s}^{\delta}$ against an alternative hypothesis $H_{1}: p_{s}<\gamma_{s}^{\delta}$. Let $\overline{X_{s}^{U}}$ define the average sample value $X_{s}^{U}$, we use $\gamma_{s}^{\delta}-\overline{X_{s}^{U}}$ as a test statistic, rejecting the null hypothesis if $\gamma_{s}^{\delta}-\overline{X_{s}^{U}}$ is too large. Accordingly, the standardized $z$-statistics for $s$ state is defined by the formula:

$$
z_{s}=\frac{\gamma_{s}^{\delta}-\overline{X_{s}^{U}}}{\sqrt{\frac{\gamma_{s}^{\delta}\left(1-\gamma_{s}^{\delta}\right)}{n_{s}}}} .
$$

Since we did not observe the transition $\delta$, i.e. $\overline{X_{S}^{U}}=0$, from the preceding equation it follows that

$$
z_{s}=\frac{\gamma_{s}^{\delta}}{\sqrt{\frac{\gamma_{s}^{\delta}\left(1-\gamma_{s}^{\delta}\right)}{n_{s}}}} .
$$

Statistical normal distribution statistics, $z_{\alpha}$ with $\alpha$ certainty can be found in the statistical textbooks [15]. If $z_{s}<z_{\alpha}$, we accept $H_{0}$, and we need to collect more data. Otherwise, we say that sufficient data was collected. The following two statements show that $z$ test should be performed only one time for all states.

Statement 1. For $z$-statistics data $z_{1}$ and $z_{2}$ statistics $\min \left\{z_{1}, z_{2}\right\}$ can be compared with a reference $z$-statistics instead of testing $z_{1}$ and $z_{2}$ separately.

Proof. Let's assume, that $\gamma_{s}^{\delta}$ remains constant for the experiment and is equal for $z_{1}$ and $z_{2}$. From equation (27) $z_{s} \alpha \sqrt{n_{s}}$. Without loss of generality, we assume that $z_{1}=\min \left\{z_{1}, z_{2}\right\}$. As $n_{s} \in N^{+}$and $\sqrt{n_{s}}>1$, then in order to $z_{1}<z_{2}$ be performed, $n_{1}<n_{2}$. The purpose of the comparison with the reference statistics is to find the amount of data required for a certain level of trust. If $z_{1}<z_{\alpha}$, then, consequently, $z_{2}<z_{\alpha}$. If $z_{1}$ requires more data to meet this condition, minimum $z_{1}$ and $z_{2}$ satisfies both the requirements of statistics and the requirements of the application.

Statement 2. For $z$-statistics for all states $\left\{z_{1}, z_{1}, \ldots, z_{|S|}\right\}$, define $z_{\text {exp }}$ as:

$$
z_{\text {exp }}=\min _{s} z_{s}
$$

To define whether the current model is statistically sufficient for detection of an outbound transition with a probability of occurrence $\varepsilon, z$-test should be performed only once, using $z_{\text {exp }}$ as test statistics.

Proof. Proof is a simple continuation of the proof 1 , in which more than two $z$-statistics are recorded.

If we can not reject the null hypothesis for all states, then we need to estimate how much preparation data must be collected for reconstruction. Let's assume, the model state structure constructed from $y^{D}$, complete with a respect to the underlying process, we can easily calculate the minimum amount of training data $D^{\alpha}$, necessary to reject the null hypothesis $H_{0}$, having solved the right hand side of equation (27) equal to $z_{\alpha}$. It provides

$$
\frac{\gamma_{s}^{\delta}}{\sqrt{\frac{\gamma_{s}^{\delta}\left(1-\gamma_{s}^{\delta}\right)}{n_{s}}}}=z_{\alpha}
$$

Solution for $n_{s}$ in terms $\gamma_{s}^{\delta}$ and $z_{\alpha}$ gives

$$
n_{s}=\frac{z_{\alpha}^{2}\left(1-\gamma_{s}^{\delta}\right)}{\gamma_{s}^{\delta}} \text {. }
$$

Then follows:

$$
D^{\alpha}=\frac{n_{s}}{\pi_{s}} .
$$

That is why it is necessary for us to extend the sequence of training data, having collected $D^{\alpha}-D$ more observations.

Definition. Using the calculation for a family error [6], the confidence of the model can be determined as:

$$
\alpha_{f}=1-\prod_{s \in S}\left(1-P\left(Z<z_{s}\right)\right),
$$

where $z_{S}$ is statistics, calculated for states using equations (27), and $P\left(Z<z_{s}\right)$ is a probability of the fact, that normal distribution has a value, less than $z_{s}$.

\section{Conclusions}

In this work the problem, arising at constructing or study of HMM class of observation data is analyzed and solved, which allows precisely to define both data, used for model training and the underlying process. Process statistics was used for calculating of the upper limit of the necessary samples number to ensure that the model has a given significance level. 


\title{
References
}

1. The official site of The Tor Project, available at: www.torproject.org/ (accessed 8 November 2020).

2. The official site of The JAP: Anonymity and privacy. www.anon.inf.tu-dresden.de/ (accessed 8 November 2020).

3. The official site of The I2p anonymous network. www.geti2p.net/en. (accessed 8 November 2020).

4. Ye Zhu, Xinwen Fu, Graham, B., Bettati, R. and Wei Zhao. (2010). Correlation-Based Traffic Analysis Attacks on Anonymity Networks. IEEE Transactions on Parallel and Distributed Systems, 21(7), 954-967. doi:10.1109/tpds.2009.146.

5. Acquisti, A., Dingledine, R. and Syverson, P. (2003). On the Economics of Anonymity. Lecture Notes in Computer Science, 84-102. https://doi.org/10.1007/978-3-540-45126-6_7.

6. Buttyan, L. and Holczer, T. (2012). Traffic analysis attacks and countermeasures in wireless body area sensor networks. 2012 IEEE International Symposium on a World of Wireless, Mobile and Multimedia Networks (WoWMoM). https://doi.org/10.1109/wowmom.2012.6263774.

7. Shalizi, C.R., Shalizi, K.L. and Crutchfield, J.P. (2002). An algorithm for pattern discovery in time series. Arxiv preprint cs/0210025.

8. McHugh, J., Christie, A. and Allen, J. (2000). Defending Yourself: The Role of Intrusion Detection Systems. IEEE Software, 17(5), 42-51. https://doi.org/10.1109/52.877859.

9. Moriasi, D.N., Arnold, J.G., Van Liew, M.W., Bingner, R.L., Harmel, R.D. and Veith, T.L. (2007). Model Evaluation Guidelines for Systematic Quantification of Accuracy in Watershed Simulations. Transactions of the ASABE, 50(3), 885-900. https://doi.org/10.13031/2013.23153.

10. Rosset, S., Perlich, C. and Zadrozny, B. Ranking-Based Evaluation of Regression Models. Fifth IEEE International Conference on Data Mining (ICDM'05). https://doi.org/10.1109/icdm.2005.126.

11. Samuels, J.D. and Sekkel, R.M. (2017). Model Confidence Sets and forecast combination. International Journal of Forecasting, 33(1), 48-60. https://doi.org/10.1016/j.ijforecast.2016.07.004.

12. Rajgopal, J. and Mazumdar, M. (2002). Modular operational test plans for inferences on software reliability based on a Markov model. IEEE Transactions on Software Engineering, 28(4), 358-363. https://doi.org/10.1109/tse.2002.995424.

13. Schwier, J.M., Brooks, R.R., Griffin, C. and Bukkapatnam, S. (2009). Zero knowledge hidden Markov model inference. Pattern Recognition Letters, 30(14), 1273-1280. https://doi.org/10.1016/j.patrec.2009.06.008.

14. Brooks, R.R., Schwier, J.M. and Griffin, C. (2009). Behavior Detection Using Confidence Intervals of Hidden Markov Models. IEEE Transactions on Systems, Man, and Cybernetics, Part B (Cybernetics), 39(6), $1484-1492$. https://doi.org/10.1109/tsmcb.2009.2019732.

15. Bowerman, B.L. and O'Connell, R.T. (1992). Linear Statistical Models: An Applied Approach. Biometrics, $48(1), 333$. https://doi.org/10.2307/2532769.

\section{Відомості про автора:}

\section{Hyx Taxa Haciф}

кандидат наук

завідувач кафедри

Університетського коледжу AL-Nukhba, Багдад, Ірак

https://orcid.org/0000-0001-9137-9914
Information about the author:

Nooh Taha Nasif

Doctor of Philosophy

Head of Department

of AL-Nukhba University College,

Baghdad, Iraq

https://orcid.org/0000-0001-9137-9914

\section{ВИКОРИСТАННЯ СТАТИСТИЧНОГО АНАЛІЗУ ТРАФІКУ ДЛЯ ОЦІНКИ БЕЗПЕКИ ПЕРЕДАЧІ ІНФОРМАЦІЇ}

\author{
Hyx Taxa Haciф
}

У статті на основі моделювання стохастичних систем проводиться аналіз проблем безпеки в мережі Інтернет. Такий підхід дозволяє виявити недоліки в існуючих системах безпеки та запропонувати нові рімення захисту від кібератак. 3 цією метою розглянуті $і$ запропоновані нові, гнучкі та адаптивні схеми забезпечення безпеки. Зокрема запропонована гібридна схема мережевої безпеки, яка включає в себе як системи виявлення атак, так і пасток для кібератак. При иьому мережева система безпеки на основі активності є логічним доповненням систем пасивного виявлення з використанням IDS. Запропонована децентралізація процесу прийняття рімень Маркова (DEC-РОМDР) дозволяє добре збалансувати показники безпеки, які будуть забезпечені, з необхідними витратами.

Ключові слова: безпека в мережі, системи виявлення атак, аналіз трафіку.

\section{ИСПОЛЬЗОВАНИЕ СТАТИСТИЧЕСКОГО АНАЛИЗА ТРАФИКА ДЛЯ ОЦЕНКИ БЕЗОПАСНОСТИ ПЕРЕДАЧИ ИНФОРМАЦИИ}

\author{
Нух Таха Насиф
}

В статье на основе моделирования стохастических систем проводится анализ проблем безопасности в сети Интернет. Такой подход позволяет выявить недостатки в существующих системах безопасности и предложить новые решения защиты от кибератак. С этой целью рассмотрены и предложены новые, гибкие и адаптивные схемы обеспечения безопасности. В частности предложена гибридная схема сетевой безопасности, которая включает в себя как системы обнаружения атак, так и ловушек для кибератак. При этом сетевая система безопасности на основе активности является логическим дополнением систем пассивного обнаружения с использованием IDS. Предложенная децентрализация процесса принятия решений Маркова (DEC-PОМDP) позволяет хорошо сбалансировать показатели безопасности, которые будут обеспечены, с необходимыми затратами.

Ключевые слова: безопасность в сети, системы обнаружения атак, анализ трафика. 